\title{
Substance Use Dose Frequency
}

National Cancer Institute

\section{Source}

National Cancer Institute. Substance Use Dose Frequency. NCI Thesaurus. Code C83044.

The rate of substance usage. 